\title{
Possible exit channel effect on isomer yield ratios
}

\author{
M. Kildir,* Z. Morel,* Z. Büyükmumcu,* H. N. Erten** \\ *Department of Chemistry, Middle East Technical University, Ankara, Turkey \\ **Department of Chemistry, Bilkent University, Ankara, Turkey
}

(Received February 3, 1997)

\begin{abstract}
Isomer yield ratio measurements in fission are important in understanding the fission process. With the development of new instrumental techniques, a large number of yield data are now available. The experimental data on isomer yield ratios in the thermal neutron induced fission of ${ }^{235} \mathrm{U}$ are compared with those calculated from the simple statistical model by MADLAND and ENGLAND. The method of calculation has been extended to the isotopes having more than one isomeric state. The results may be explained according to the multi-exit-channel model of fission.
\end{abstract}

\section{Introduction}

Extensive experimental data reflecting various aspects of the fission process have been reported for different fissioning systems. Among them, the angular momentum of primary fission fragments is of both experimental and theoretical interest. The total angular momentum of a fissioning nucleus just before separation is distributed between the orbital and the intrinsic angular momentum of the fission fragments. Isomer yield ratio measurements in fission provide information about the intrinsic angular momentum. Several isomer ratios have been measured for thermal neutron fission. ${ }^{1-8}$ The most extensive study of isomer yields in the thermal neutron fission of ${ }^{235} U$ has been carried out by RUDSTAM et al. ${ }^{7}$ using an on line isotope separator.

MADLAND and ENGLAND 9 have developed a simple statistical model for calculating isomer yield ratios of products formed in neutron induced fission. They assumed that the fission fragments are formed with a density distribution, $P(I)$, of total angular momentum, $J$ which is characterized by the parameter, $J_{\text {ms }}=\left(\left\langle J^{2}\right\rangle\right)^{1 / 2}$. Here, the branching is simply assumed to be the result of the competition of isomers of different spins for the fragments of various intrinsic angular momentum. The parameter, $J_{\mathrm{ms}}$, which determines the spin distribution is taken to be constant for all fragment masses in the thermal neutron induced fission of all actinide systems. Much work has been devoted in recent years along the line of the multi-exit-channel model of fission as elaborated by Brosa, Grossmann and Muller ${ }^{10}$ (BGM-model). The obvious feature of BGM-model is the variation of $J_{\text {rms }}$ as a function of the primary fragment.

In this work the experimental data on isomer yield ratios in the thermal neutron induced fission of ${ }^{235} \mathrm{U}$ have been compiled and compared with those calculated with the recipe by MadLAND and England. 9 The formulation of an extended recipe for nuclei which contains two isomeric states is also presented. The results are qualitatively interpreted along the predictions of BGM-model.

\section{Calculations}

The isomer yield ratios of products with only one isomeric state formed in the thermal neutron induced fission of ${ }^{235} \mathrm{U}$ can be calculated by the simple statistical model by MADLAND and ENGLAND. ${ }^{9}$ We present below the extension of this model to nuclei which have two isomeric states. Using similar notation as the original paper, suppose we have such a nucleus with intrinsic angular momentum (or spin) values indicated by $J_{h}, J_{m}$ and $J_{l}$ where $h$ and $l$ is used for the highest and lowest values of the spins and $m$ is for the intermediate spin. We do not differentiate the ground state spin but an appropriate one could be taken as the ground state and indicated by the symbol $g$. The branching ratio is obtained using the angular momentum density distribution as given by:

$$
P(J)=P_{0}(2 J+1) \exp \left[-(J+1 / 2)^{2} /\left\langle J^{2}\right\rangle\right]
$$

where $J_{r m s}=\left(\left\langle J^{2}\right\rangle\right)^{1 / 2}$, characterizes the angular momentum of the initial fragment. The isomer yield ratios can be obtained by:

$\frac{I Y(h)}{I Y(h)+I Y(m)+I Y(l)}=\frac{\int_{J_{h m}}^{\infty} P(J) \mathrm{d} J}{\int_{0 \text { or } 1 / 2}^{\infty} P(J) \mathrm{d} J}$
$\frac{I Y(m)}{I Y(h)+I Y(m)+I Y(l)}=\frac{\int_{J_{m t}}^{\infty} P(J) \mathrm{d} J-\int_{J_{m m}}^{\infty} P(J) \mathrm{d} J}{\int_{0 \text { or } 1 / 2}^{\infty} P(J) \mathrm{d} J}$

$$
\frac{I Y(l)}{I Y(h)+I Y(m)+I Y(l)}=\frac{1-\int_{J_{m l}}^{\infty} P(J) \mathrm{d} J}{\int_{0 \text { or } 1 / 2}^{\infty} P(J) \mathrm{d} J}
$$

There result eight separate cases in calculating isomer yield ratios using Eqs (2), (3) and (4) depending on 
Table 1. Isomer ratio equations in terms of $F$ functions for nuclei with more than one isomeric state. The $F$ function is defined as by MADLAND and ENGLAND ${ }^{9}$

\begin{tabular}{|c|c|c|c|c|}
\hline \multicolumn{5}{|l|}{ Odd-A } \\
\hline$J_{h}-J_{m}$ & Even & Odd & Even & Odd \\
\hline$J_{m}-J_{l}$ & Even & Odd & Odd & Even \\
\hline$\alpha\left(J_{h}\right)$ & $F_{1}^{h m}$ & $F_{2}^{h m}$ & $F_{1}^{h m}$ & $F_{2}^{h m}$ \\
\hline$\overline{\sigma\left(J_{m}\right)}$ & $F_{1}^{m l}-F_{1}^{h m}$ & $F_{2}^{m l}-F_{2}^{h m}$ & $F_{2}^{m I}-F_{1}^{h m}$ & $F_{1}^{m}-F_{2}^{h m}$ \\
\hline$\sigma\left(J_{h}\right)$ & $F_{1}^{h m}$ & $F_{2}^{h m}$ & $F_{1}^{h m}$ & $F_{2}^{h m}$ \\
\hline$\sigma\left(J_{l}\right)$ & $1-F_{1}^{m l}$ & $\overline{1-F_{2}^{m I}}$ & $1-F_{2}^{m I}$ & $1-F_{1}^{m l}$ \\
\hline \multicolumn{5}{|l|}{ Even-A } \\
\hline$J_{h}-J_{m}$ & Even & Odd & Even & Odd \\
\hline$J_{m}-J_{l}$ & Even & Odd & Odd & Even \\
\hline$\sigma\left(J_{h}\right)$ & $F_{3}^{h m}$ & $F_{4}^{h m}$ & $F_{3}^{h m}$ & $F_{4}^{h m}$ \\
\hline$\overline{\sigma\left(J_{m}\right)}$ & $F_{3}^{m l}-F_{3}^{h m}$ & $F_{4}^{m l}-F_{4}^{h m}$ & $F_{4}^{m l}-F_{3}^{h m}$ & $F_{3}^{m l}-F_{4}^{h m}$ \\
\hline$\sigma\left(J_{h}\right)$ & $F_{3}^{h m}$ & $F_{4}^{h m}$ & $F_{3}^{h m}$ & $F_{4}^{h m}$ \\
\hline$\overline{\sigma\left(J_{l}\right)}$ & $1-F_{3}^{m l}$ & $1-F_{4}^{m l}$ & $1-F_{4}^{m I}$ & $1-F_{3}^{m l}$ \\
\hline
\end{tabular}

whether the fission product mass number $A, \Delta J_{h m}=J_{m}$ and $\Delta J_{m l}=J_{m}-J_{l}$ is even or odd. These cases are easily composed using the $F$ functions of MADLAND and ENGLAND. ${ }^{9}$ For odd- $A$ nuclei: $F_{1}$ and $F_{2}$ functions are used for even or odd values of $\Delta I_{h m}\left(\Delta J_{m l}\right)$, respectively. The $F_{3}$ and $F_{4}$ functions are similarly used instead for even- $A$ nuclei. In order to keep track of which spin pair is used in the calculations, the $F$ function is given a superscript of either $\mathrm{hm}$ or $\mathrm{ml}$. As an example $F_{1}^{\mathrm{hm}}$ indicates that the $F$ function is calculated for an odd- $A$ nucleus in which $\Delta J_{h m}$ has an even value. The isomer ratio equations in terms of $F$ functions are summarized in Table 1 for the eight possible cases.

\section{Results and discussion}

The experimental isomer yield ratios of four indium isotopes each having two isomeric states from RUDSTAM et al., ${ }^{7}$ as well as those calculated using the above prescription, are given in Table 2 . The calculated results are in reasonably good agreement with the experimental values for the three isotopes of ${ }^{120} \mathrm{In},{ }^{122} \mathrm{In},{ }^{130} \mathrm{In}$. However, for ${ }^{131}$ In the calculated isomer ratio is much larger than the experimental one, probably indicating that the $J_{r m s}$ is much smaller than the assumed value of $7.0 \pm 0.5$. Since, this isotope has the magic number of 82 neutrons, the fragment has a large resistance to deformation and may assume a prescission shape with very small deformation. Similar expectation for ${ }^{130} \mathrm{In}$ with $N=81$ may be diminished due to the valence $p$ - $n$ interaction in the $N_{p} N_{n}$ scheme ${ }^{11}$ which enhances deformability of the nucleus even around the ground state. This is not the total $p-n$ interaction but the deformation-driving part of it which is primarily the $T=0$ component. We may indicate here that the isomer ratios for

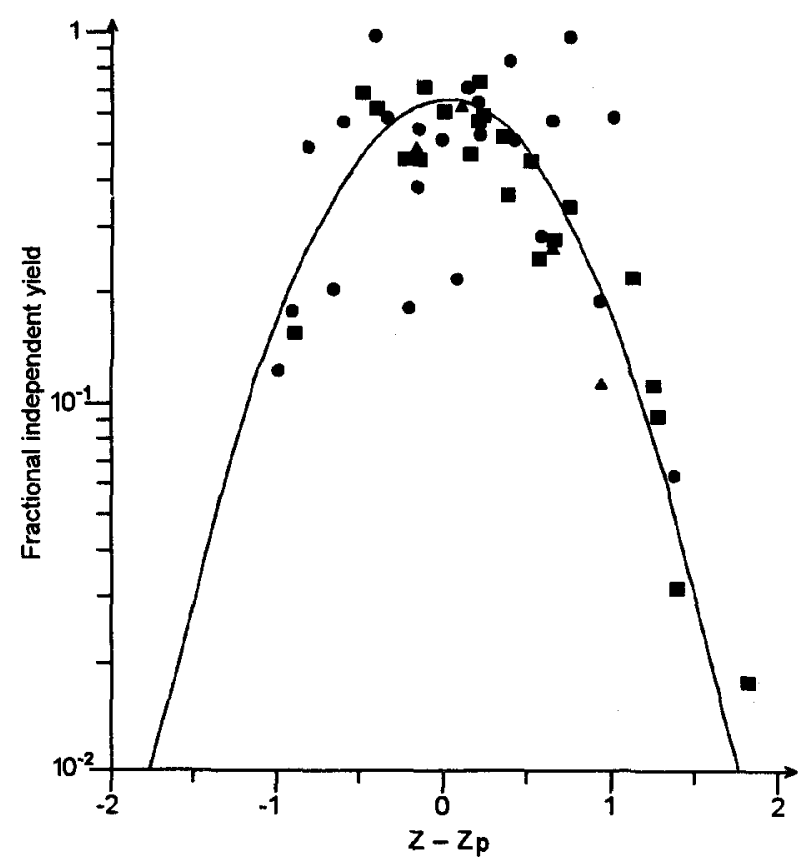

Fig. 1. Experimental fractional independent yields of the isomers considered in Table 1 . Full circles are from RuDstaM et al. ${ }^{7}$ The squares are from the literature ${ }^{6,12-18}$ and the triangles are from ERTEN. ${ }^{8}$

The curve is the normal charge distribution curve from systematics using the extended $Z_{p}$ model of $W_{A H L}$ with $\sigma_{Z}=0.531$ and even-odd neutron and proton factors set as 1

${ }^{120}$ In and ${ }^{122}$ In calculated with $J_{r m s}=9.0 \pm 0.5$ give better agreement with the experimental values, whereas for ${ }^{130} \mathrm{I}$ better agreement is obtained with the use of $J_{r m s}=7.0 \pm$ \pm 0.5 .

Figure 1 shows the experimental fractional independent yields of isomers in the thermal neutron 
M. KILDIR et al.: POSSIBLE EXTT CHANNEL ERFECT ON ISOMER YIELD RATIOS

Table 2. Experimental and calculated isomer ratios of indium isotopes having two isomeric states. Calculations were done using equations given in Table 1

\begin{tabular}{|c|c|c|c|c|c|c|c|c|c|c|c|c|c|}
\hline \multirow{3}{*}{ Isotope } & \multirow{3}{*}{$Z-Z_{p}$} & \multirow{2}{*}{\multicolumn{3}{|c|}{ Spin/parity }} & \multirow{2}{*}{\multicolumn{3}{|c|}{ Half-life, s }} & \multirow{2}{*}{\multicolumn{2}{|c|}{$\frac{\begin{array}{c}\text { Isomeric yield ratio } \\
\text { (experimental) }\end{array}}{\text { RUDSTAM et al. }{ }^{9}}$}} & \multicolumn{4}{|c|}{$\begin{array}{l}\text { Isomeric yield ratio } \\
\text { (calculated) }\end{array}$} \\
\hline & & & & & & & & & & \multicolumn{2}{|c|}{$\left\langle J^{2}\right\rangle^{1 / 2}=7.0 \pm 0.5$} & \multicolumn{2}{|c|}{$\left\langle J^{2}\right\rangle^{1 / 2}=9.0 \pm 0.5$} \\
\hline & & $h$ & $m$ & $l$ & $h$ & $m$ & 1 & $\sigma_{h} / \sigma_{l}$ & $\sigma_{h} / \sigma_{m}$ & $\sigma_{h} / \sigma_{l}$ & $\sigma_{h} / \sigma_{m}$ & $\sigma_{h} / \sigma_{l}$ & $\sigma_{h} / \sigma_{m}$ \\
\hline${ }^{120} \mathrm{In}$ & +1.374 & $8^{-}$ & $5^{+}$ & $1^{+}$ & 47.3 & 46.2 & 3.08 & $0.74 \pm 1.45$ & $1.5 \pm 0.8$ & $0.84-1.48$ & $0.63-0.97$ & $2.3-3.4$ & $1.4-1.8$ \\
\hline${ }^{122} \mathrm{In}$ & +0.569 & $8^{-}$ & $5^{+}$ & $1^{+}$ & 10.0 & 10.5 & 1.5 & $1.7 \pm 2.5$ & $2.0 \pm 0.5$ & $0.84-1.48$ & $0.63-0.97$ & $2.3-3.4$ & $1.4-1.8$ \\
\hline${ }^{130} \mathrm{In}$ & -1.358 & $10^{-}$ & $5^{+}$ & 1 & 0.53 & 0.53 & 0.33 & $0.78 \pm 0.17$ & $0.57 \pm 0.14$ & $0.36-0.58$ & $0.57-1.11$ & $1.8-2.7$ & $0.86-1.17$ \\
\hline${ }^{131}$ In & -1.769 & $21 / 2^{+}$ & $9 / 2^{+}$ & $1 / 2^{-}$ & 0.32 & 0.28 & 0.35 & $\begin{array}{l}2.4 \cdot 10^{-2} \pm \\
\pm 9.0 \cdot 10^{-3}\end{array}$ & $\begin{array}{l}4.8 \cdot 10^{-3} \pm \\
\pm 2.4 \cdot 10^{-3}\end{array}$ & $0.75-1.47$ & $0.31-0.52$ & $2.5-3.7$ & $0.80-1.08$ \\
\hline
\end{tabular}

Table 3. Experimental and calculated isomer ratios of products in thermal neutron fission of ${ }^{235} \mathrm{U}$ calculated according to the model of MADLAND and ENGLAND 9

\begin{tabular}{|c|c|c|c|c|c|c|c|c|c|c|}
\hline \multirow{2}{*}{ Isotope } & \multirow{2}{*}{$Z-Z_{p}$} & \multicolumn{2}{|c|}{ Spin/parity } & \multicolumn{2}{|c|}{ Half-life, s } & \multicolumn{3}{|c|}{ Isomeric yield ratio (experimental) } & \multicolumn{2}{|c|}{ Isomeric yield ratio (calculated) } \\
\hline & & $m$ & $g$ & $m$ & $g$ & RUDSTAM et al. ${ }^{7}$ & $\mathrm{UKFY}^{6}$ & $\operatorname{ERTEN}^{8}\left\langle J^{2}\right\rangle$ & ${ }^{2} \gamma^{1 / 2}=7.0 \pm 0.5$ & $\left\langle J^{2}\right\rangle^{1 / 2}=9.0 \pm 0.5$ \\
\hline${ }^{79} \mathrm{Ge}$ & +0.354 & $7 / 2^{+}$ & $1 / 2^{-}$ & 39.0 & 18.4 & $3.6 \pm 20$ & & & $4.8-6.5$ & \\
\hline${ }^{81} \mathrm{Ge}$ & -0.454 & $1 / 2^{+}$ & $9 / 2^{+}$ & 7.5 & 7.6 & $0.44 \pm 0.12$ & & & $0.32-0.23$ & \\
\hline${ }^{83} \mathrm{Se}$ & +0.738 & $1 / 2^{-}$ & $9 / 2^{+}$ & 70.4 & 1350 & $0.13 \pm 0.09$ & & & $0.32-0.23$ & \\
\hline${ }^{90} \mathrm{Rb}$ & -0.919 & 4 & $1^{-}$ & 251 & 153 & $1.4 \pm 0.3$ & & & $3.0-4.2$ & \\
\hline${ }^{92} \mathrm{Nb}$ & +4.113 & $2^{+}$ & $7^{+}$ & $5.26 \cdot 10^{6}$ & $1.10 \cdot 10^{15}$ & & $1.61 \pm 1.07$ & & $1.03-0.70$ & \\
\hline${ }^{97} \mathrm{Nb}$ & +2.138 & $1 / 2^{-}$ & $9 / 2^{+}$ & 58.1 & 4417 & & $0.44 \pm 0.16$ & & $0.32-0.23$ & \\
\hline${ }^{102} \mathrm{Rh}$ & +4.209 & $6^{+}$ & $\left(1^{-}, 2\right)$ & $-2.9 y$ & $7.452 \cdot 10^{6}$ & & $0.80 \pm 0.44$ & & $\begin{array}{l}(1.3-1.8)^{2-} \\
(1.6-2.3)^{1-}\end{array}$ & \\
\hline${ }^{115} \mathrm{Ag}$ & +1.399 & $7 / 2^{+}$ & $12^{-}$ & 18 & 388.6 & & $2.2 \pm 1.2$ & & $4.8-6.5$ & $8.5-10.8$ \\
\hline${ }^{117} \mathrm{Ag}$ & +0.583 & $7 / 2^{+}$ & $1 / 2^{-}$ & 5.34 & 73 & & $11.7 \pm 3.4$ & & $4.8-6.5$ & $8.5-10.8$ \\
\hline${ }^{118} \mathrm{Ag}$ & +0.171 & 6 & $3^{+}$ & 2.8 & 3.7 & $0.79 \pm 0.32$ & & & $0.97-1.4$ & $1.9-2.5$ \\
\hline${ }^{120} \mathrm{Ag}$ & -0.626 & 6 & $3^{+}$ & 0.32 & 1.17 & $5.6 \pm 6.4$ & & & $0.97-1.4$ & $1.9-2.5$ \\
\hline${ }^{121} \mathrm{Cd}$ & -0.042 & $11 / 2^{-}$ & $1 / 2^{-1}$ & 8.3 & 12.5 & $8.3 \pm 10$ & & & $2.3-3.3$ & $4.3-5.5$ \\
\hline${ }^{123} \mathrm{In}$ & +0.184 & $1 / 2^{-}$ & $9 / 2^{+}$ & 45.9 & 6.68 & $0.064 \pm 0.119$ & & & $0.32-0.23$ & $0.18-0.14$ \\
\hline${ }^{124}$ In & -0.226 & $8^{-}$ & $3^{+}$ & 3.69 & 3.09 & $5.8 \pm 39$ & & & $0.59-0.90$ & $1.3-1.7$ \\
\hline $125_{\mathrm{In}}$ & -0.525 & $1 / 2^{-}$ & $9 / 2^{+}$ & 12.2 & 2.33 & & $0.46 \pm 0.61$ & & $0.32-023$ & $0.18-0.14$ \\
\hline${ }^{125} \mathrm{Sn}$ & +0.475 & $3 / 2^{+}$ & $11 / 2^{-}$ & 571.2 & $8.33 \cdot 10^{5}$ & & $0.58 \pm 0.51$ & & $0.58-0.41$ & $0.31-0.24$ \\
\hline${ }^{126} \mathrm{In}$ & -0.680 & $8^{-}$ & $3^{+}$ & 1.65 & 1.60 & $0.76 \pm 0.99$ & & & $0.59-0.90$ & $1.3-1.7$ \\
\hline${ }^{127}$ In & -0.843 & $1 / 2^{-}$ & $9 / 2^{+}$ & 3.81 & 1.22 & $0.15 \pm 0.07$ & & & $0.32-0.23$ & $0.18-0.14$ \\
\hline${ }^{127} \mathrm{Sn}$ & +0.157 & $3 / 2^{+}$ & $11 / 2^{+}$ & 247.8 & 7560 & & $0.13 \pm 0.05$ & & $0.58-0.41$ & $0.31-0.24$ \\
\hline${ }^{128} \mathrm{In}$ & -1.009 & $8^{-}$ & $3^{+}$ & 0.72 & 0.84 & $0.43 \pm 0.15$ & & & $0.59-0.90$ & \\
\hline${ }^{130} \mathrm{Sb}$ & +0.642 & $8^{-}$ & $4^{+}$ & 2400 & 378 & & $1.5 \pm 0.2$ & $0.66 \pm 0.25$ & $0.48-0.74$ & \\
\hline${ }^{131} \mathrm{Te}$ & +1.231 & $11 / 2^{-}$ & $3 / 2^{+}$ & 108000 & 1500 & & $2.7 \pm 0.3$ & & $1.7-2.4$ & \\
\hline $132 \mathrm{I}$ & +1.812 & $8^{-}$ & $4^{+}$ & 5016 & 8240 & & $3.8 \pm 1.3$ & & $0.48-0.74$ & \\
\hline${ }^{132} \mathrm{Sb}$ & -0.188 & $4^{+}$ & $8^{-}$ & 168 & 252 & $3.9 \pm 0.9$ & & $2.1 \pm 0.8$ & $2.2-1.4$ & \\
\hline${ }^{133} \mathrm{Te}$ & 0.376 & $112^{-}$ & $3 / 2^{+}$ & 3325 & 746 & $3.6 \pm 0.9$ & & & $1.7-2.4$ & \\
\hline${ }^{133} \mathrm{Xe}$ & +2.376 & $112^{-}$ & $3 / 2^{+}$ & 189216 & 452995 & & $2.4 \pm 0.4$ & & $1.7-2.4$ & \\
\hline${ }^{134} I$ & +0.922 & $8^{-}$ & $4^{+}$ & 228 & 3120 & $0.25 \pm 0.21$ & & $1.4 \pm 0.7$ & $0.48 \pm 0.74$ & \\
\hline${ }^{134} \mathrm{Cs}$ & +2.922 & 8 & $4^{+}$ & 10451 & $2.0648 \mathrm{y}$ & & $1.15 \pm 0.25$ & & $0.48-0.74$ & \\
\hline${ }^{135} \mathrm{Xe}$ & +1.483 & $11 / 2^{-}$ & $3.2^{+}$ & 917 & 32904 & & $1.8 \pm 0.3$ & & $1.7-2.4$ & \\
\hline $136 I$ & +0.067 & 6 & $2^{-}()$ & 48 & 83 & $3.8 \pm 3.2$ & & $1.3 \pm 0.5$ & $1.3-1.8$ & \\
\hline${ }^{138} \mathrm{Cs}$ & +1.254 & 6 & $3^{-}$ & 174 & 2005 & & $0.98 \pm 0.16$ & & $0.97-1.4$ & \\
\hline${ }^{82} \mathrm{As}$ & +0.142 & 5 & $2-$ & 13 & 21 & $0.21 \pm 0.10$ & & & $1.6-2.3$ & \\
\hline${ }^{116} \mathrm{Ag}$ & +0.996 & $6^{+}\left(5^{+}\right)$ & $1^{+}\left(2^{-}\right)$ & 18 & 1280 & $0.22 \pm 0.18$ & & & $1.6-2.3$ & \\
\hline${ }^{129} \mathrm{Sn}$ & -0.176 & $11 / 2^{+}$ & $3 / 2^{+}$ & 534 & 134 & $0.75 \pm 0.17$ & & & $1.7-2.1$ & \\
\hline${ }^{130} \mathrm{Sn}$ & -0.258 & 7 & $0^{+}$ & 102 & 222 & $0.14 \pm 0.03$ & & & $1.6-2.3$ & \\
\hline${ }^{133} \mathbf{I}$ & +1.376 & $19 / 2^{-}$ & $7 / 2^{+}$ & 9.0 & 74880 & & $0.07 \pm 0.02$ & & $0.38-0.61$ & \\
\hline
\end{tabular}




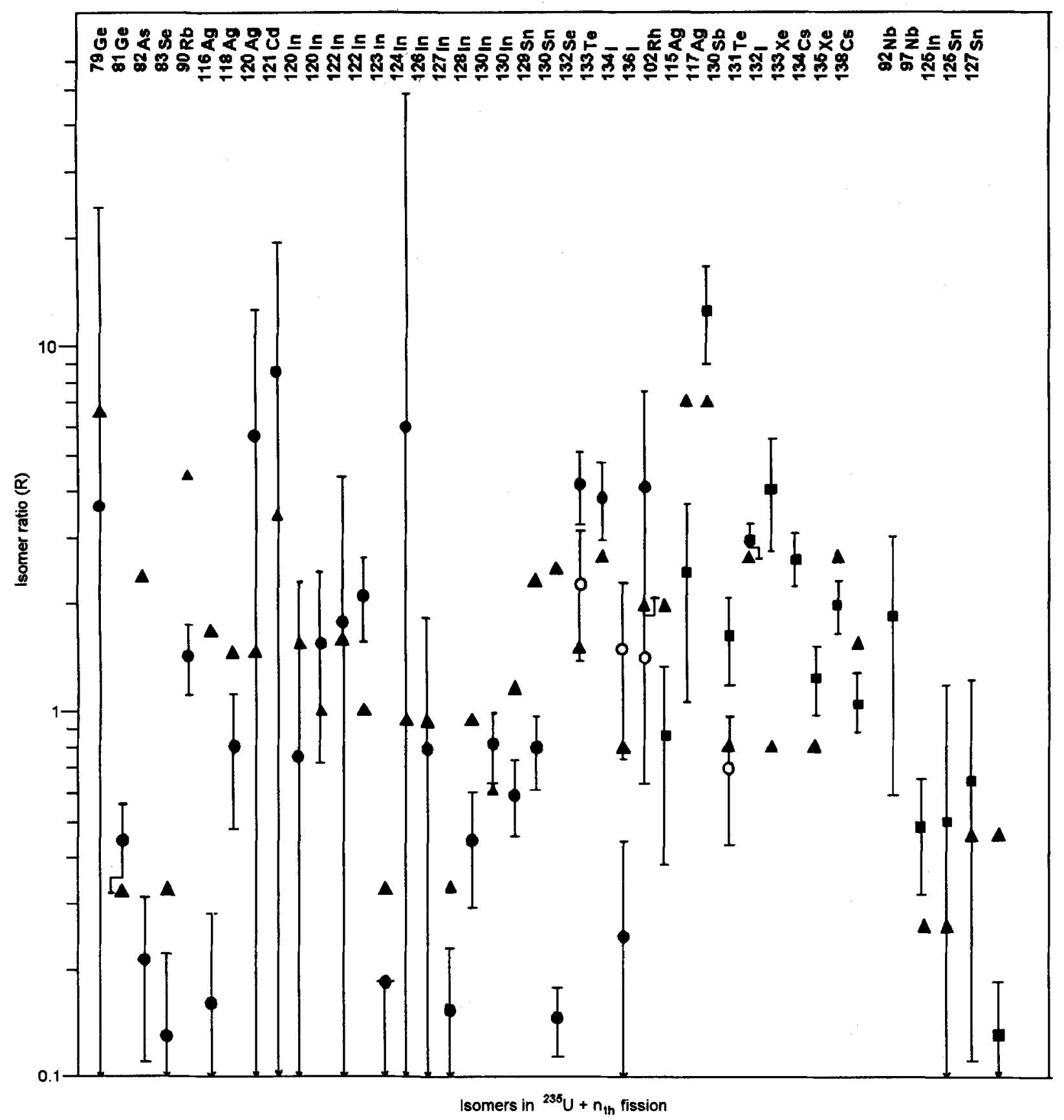

Fig. 2. Comparison of experimental and calculated isomer ratios, $\mathrm{R}\left(I^{m} / T^{\mathrm{g}}\right)$ in the thermal neutron fission of ${ }^{235} \mathrm{U} ; \Delta$ calculated values assuming a $J_{r m s}$ of $7.5 ;-$ experimental data from RuDsTAM et al. ${ }^{7} ; O$ experimental data from ERTEN; ${ }^{8}$ experimental data from Refs $6,12-18$

fission of ${ }^{235} \mathrm{U}$ and the charge distribution curve from the $Z_{p}$ model of WAHL. ${ }^{12}$ Experimental errors were not indicated in order not to complicate the figure. It is seen that most of the experimental yields follow Gaussian curve. There are, however, some notable exceptions, particularly in the data of RuDstam et al. ${ }^{7}$ We believe that the data reported for ${ }^{81} \mathrm{Ge},{ }^{83} \mathrm{Se}$ and ${ }^{133} \mathrm{Te}$ are probably cumulative yields. The values for ${ }^{116} \mathrm{Ag},{ }^{120} \mathrm{Ag}$, ${ }^{127}$ In are too high, whereas the values for ${ }^{124} \mathrm{In},{ }^{126} \mathrm{In}$, ${ }^{132} \mathrm{Sb}$ and ${ }^{136} \mathrm{I}$ are too low. Even, if some of the isotopic independent yields did not fit a Gaussian distribution, possibly due to calibration problems, it is believed that useful isomeric ratio information may still be obtained from these measurements.

The experimental and calculated isomer yield ratios of the nuclei with one isomeric state are given in Table 3. Calculations were done for two different values of $\left\langle\beta^{1 / 2}\right.$. Ranges were calculated in each case using the reported uncertainty. Generally, within experimental errors, the calculated values are consistent with the experimental 
results as shown in Table 3. The nuclei in which there is an order of magnitude difference between the calculated and experimental values of isomer ratios are given at the end of the table starting with ${ }^{82} \mathrm{As}$. Two of these nuclei, ${ }^{129} \mathrm{Sn}$ and ${ }^{130} \mathrm{Sn}$ have the magic proton number of $Z=50$. The ${ }^{82} \mathrm{As}$ nucleus with $N=49$ is one neutron away and the ${ }^{133}$ I nucleus with $N=80$ is two neutrons away from the corresponding magic numbers indicating that their $J_{r m s}$ values are expected to be much smaller than that of $7.0 \pm$ \pm 0.5 .

Figure 2 shows a comparison of the calculated and experimental isomeric state/ground state independent yield ratios $R$ in the thermal neutron fission of ${ }^{235} \mathrm{U}$ using all the available data in the literature. ${ }^{6-8,12-18}$ In some of the experimental results, particularly in those of RUDSTAM et al., ${ }^{7}$ the large experimental errors make a meaningful comparison with the calculated values difficult. In most cases, however, the experimental values are in reasonably good agreement with the calculated ones.

Even though quite large errors in some of the experimental results discourage us from putting forward a stronger conclusion, we would like to propose that there may be a signature of BGM-model in the results presented. Three exit channels, namely superlong (SL) and two asymmetric (S1 and S2) are predicted for the fission of ${ }^{236} \mathrm{U}$. The SL channel has a symmetric mass distribution around the mass of 118. S1 which is one of the standard channels has smaller asymmetry than that of S2. According to the picture of RASMUSSEN et al., ${ }^{19}$ the average angular momentum, $J_{a v} \cong \sqrt{\pi / 2} J_{r m s}$, is related to the prescission bending amplitude $(\gamma)$ as:

$$
J_{a v}=\frac{\sqrt{\pi}}{2 \gamma}-\frac{1}{2}
$$

The bending amplitude $(\gamma)$ or the angular positional uncertainty is in turn related to the neck radius $(r)$ and semi-major axis (a) for a fragment for a given fission channels as:

$$
\gamma=\frac{r}{a}
$$

If the parameters of $\mathrm{FAN}^{20}$ are used to calculate the average angular momentum of primary fragments as a function of their mass and exit channel the following qualitative features become apparent:

(a) $J_{a v}$ due to zero-point bending vibrations increases with an increase in the fragment mass for the SL channel. It is expected to be smaller and larger than that of $S 1$ and $S 2$ for the light and heavy fragment group, respectively.

(b) $J_{a v}$ shows saw-tooth structure for the S1 and S2 channels. The more symmetric channel (S1) gives smaller and larger values of $J_{a v}$ with respect to $S 2$ for light and heavy fragment groups, respectively.
In Tables 2 and 3 we observe that higher spin isomeric states are favorably populated for the fragments masses $116<A<126$, whereas lower spin isomeric states are favored for masses outside of this range. So there is a change in the trend around fragment mass of 127 . In order to show this trend, we have calculated isomer yield ratios of nuclei of masses $116<A<126$ using $J_{r m s}=9.0 \pm 0.5$. The agreement between the calculated values and the experimental results has improved in this mass region. Large values of $J_{r m s}$ for the fragments masses of $116<A<$ $<126$ may be indicating the contribution of highly deformed fragments of the SL channel. A similar effect was observed and interpreted in the literature, as a result of the strong influence of the spherical 82 neutron and deformed 66 neutron shells on the scission configuration. ${ }^{21}$ We now suggest that the effect may be due to the increased contribution of the SL channel as we approach symmetric division from heavy fragment side. ${ }^{22}$ Due to quite large uncertainties in the experimental results, a stronger conclusion cannot be put forward. It is clear that a definite conclusion would necessitate more precise and comprehensive data on isomer yield ratios.

\section{References}

1. I. F. Croal, H. H. Wilus, J. Inorg. Nucl. Chem., 24 (1962) 221.

2. I. F. Croal, H. H. WIILIS, J. Inorg. Nucl. Chem., 24 (1963) 1213

3. M. Imanish, I. Fujwara, T. Nishn, Nucl. Phys., A263 (1976) 141.

4. W. W. Parsons, H. D. Sharma, J. Inorg. Nucl. Chem., 36 (1974) 2392.

5. C. Chung, A. A. Hasan, S. SahIN, Radiochim. Acta, 37 (1984) 131.

6. M. F. JaMes, R. W. MILs, D. R. Weaver, Rept. AEA-TRS-1018, 1989.

7. G. Rudstam, P. Aagaard, P. Exstrom, E. Lund, H. Gokturk, H. U. SwICkY. Radiochim. Acta, 49 (1990) 155.

8. H. N. ERTEN, J. Radioanal. Nucl. Chem., 166 (1992) 187.

9. D. G. Madland, T. D. England, Nucl. Sci. Eng., 64 (1977) 859.

10. U. Brosa, S. Grossman, A. Muller, Phys. Reports, C197 (1990) 167.

11. R. F. CASTEN, Nuclear Structure from a Simple Perspective, Oxford University Press, New York, 1990.

12. A. C. WAHI, Atomic Data and Nuclear Data Tables, 39 (1988) 1.

13. G. Siegert H. Wollnik, J. Greif, R. Dicker, G. Fledler, B. PFeIfPer, Phys. Rev., C14 (1976) 1864.

14. W. Lang, H. G. Clere, H. Wohlfarth, H. Shrader, K. H. Schamid, Nucl. Phys., A345 (1980) 34.

15. H. O. Denschllag, H. Braun, W. Faubel, G. Fischbach, H. Schrader, G. Siegert, J. Blachot, Z. B. Alfassi, H. N. Erten, T. Izak-Biran, T. TAmal, A. C. Wart, K. Wolfsberg, Proc. 4th IAEA Symp. Physics and Chemistry of Fission, Jülich, Vol. 2, 1980, p. 153.

16. H. W. Weiss, N. E. Ballou, J. L. Elzie, J. M. Fresco, Phys. Rev., 188 (1969) 1893.

17. L. Robinson, A. C. WaHL, T. M. Semkow, A. G. Norris, Phys. Rev., C31 (1985) 1334.

18. T. M. Semkow, A. C. WaHL, L. Robinson, Phys. Rev., C30 (1984) 1966.

19. J. O. Rasmussen, W. Norenberg, H. J. Mang, Nucl. Phys., A136 (1969) 465.

20. Fan The-shuan, Hu Ji-Man, Bao Shang-lian, Nucl. Phys., as91 (1995) 161.

21. B. S. Tomar, A. Goswami, S. K. Das, B. K. Srivastava, R. Gunn, S. M. Sahakundu, S. Prakash, Phys. Rev., C38 (1988) 1787.

22. H. KUdo, R. SATto, M. Oda, Radiochim. Acta, 69 (1995) 145. 